\title{
Produção animal e vegetal em pastagem de Cynodon manejada sob diferentes ofertas de forragem por ovinos de dois grupos genéticos
}

\author{
Animal and vegetal production of a Cynodon pasture managed under different forage allowance by \\ wethers of two breeding groups
}

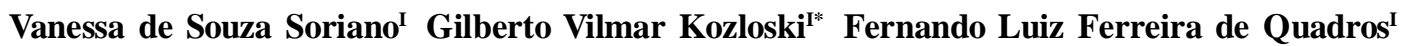 \\ Cleber Cassol Pires ${ }^{\mathrm{I}}$ Letieri Griebler $^{\mathrm{I}}$
}

\section{RESUMO}

Foi avaliado o potencial produtivo de uma pastagem de Cynodon dactylon var.dactylon manejada em um sistema de pastoreio com lotação contínua com ovinos. Testou-se o efeito de três níveis de oferta $(3,6$ ou $9 \mathrm{~kg}$ de matéria seca (MS) $100 \mathrm{~kg}^{-1}$ de peso corporal $(P C)$ ) de lâminas foliares verdes (OFLV) sobre os atributos da pastagem e sobre o desempenho de borregos de dois grupos genéticos (Texel $\times$ Ile de France e Lacaune $\times$ Texel $\times$ Ile de France $)$. O período experimental foi de 53 dias sendo utilizados 18 borregos de cada grupo genético distribuídos ao acaso em nove piquetes experimentais (três por tratamento). A OFLV real foi próxima à pretendida em cada tratamento (i.e. 3,2, 6,7 e 9,5\% do PC). A massa de forragem (média de $3474 \mathrm{~kg}$ de $M S \mathrm{ha}^{-1}$ ) teores de MS, fibra em detergente neutro e proteína bruta das amostras coletadas por simulação de pastejo (médias de 49,4, 76,1 e 12,9, respectivamente) não foram afetados pelos tratamentos. A taxa de acúmulo de lâminas foliares verdes aumentou $(P<0,05)$ de 12,1 para 20,5kg de $M S \mathrm{ha}^{-1} \mathrm{dia}^{-1}$ e a carga animal reduziu de 1178 para $609 \mathrm{~kg}$ de $\mathrm{PC} \mathrm{ha}^{-1}$ quando a OFLV aumentou de 3,2 para 9,5\% do PC. A produtividade animal por área de pastagem foi em média negativa (ganho de PC de -551 $\mathrm{g} \mathrm{ha}^{-1} \mathrm{dia}^{-1}$ ), altamente variável (coeficiente de variação de 280\%) e não foi afetada pelos tratamentos. $O$ desempenho individual dos animais foi afetado pela interação entre tratamento e grupo genético $(P<0,05)$. $O$ ganho médio diário de peso pelos ovinos cruzados com Lacaune foi positivo $\left(34 \mathrm{~g} \mathrm{dia}^{-1}\right)$ e mais alto $(P<0,05)$ na maior oferta, nulo na menor oferta e negativo $\left(-62 \mathrm{~g}\right.$ dia $\left.^{-1}\right)$ na oferta intermediária de pasto. Diferentemente, o ganho de peso dos animais Texel $\times$ Ile de France foi negativo e similar em todos os tratamentos (média de $-18 \mathrm{~g} \mathrm{dia}^{-1}$ ). Independentemente do grupo genético, o tempo diário de pastejo foi similar nos tratamentos de menor e maior oferta, mas foi menor na oferta intermediária de pasto $(P<0,05)$. Em conclusão, a pastagem de Cynodon dactylon var. dactylon apresentou um baixo potencial produtivo, principalmente quando a OFLV foi igual ou inferior a $6 \%$ do $P C$ dos animais. Contudo, os resultados indicam também que a estrutura da pastagem e o potencial genético dos animais interagem com a oferta de forragem para determinar o desempenho de animais a pasto.

Palavras-chave: Cynodon dactylon var. dactylon, comportamento ingestivo, consumo, ganho de peso, gramínea tropical, taxa de crescimento.

\section{ABSTRACT}

The productive potential of a Cynodon dactylon var.dactylon pasture managed under continuous stocking with wethers was evaluated. The effect of three $(3,6$ or $9 \mathrm{~kg}$ of dry matter (DM) $100 \mathrm{~kg}^{-1}$ of body weight $(B W)$ ) green leaf allowances $(G L A L)$ on pasture attributes and animal performance of two genetic groups $($ Texel $\times$ Ile de France and Lacaune $\times$ Texel $\times$ Ile de France) was tested. Experiment was carried out through a 53 days period with 18 wethers of each genetic group randomly distributed in nine pasture plots (three pasture plots per treatment). Actual GLAL were near to those planned for each treatment (i.e. 3.2, 6.7 e 9.5\% of BW). Forage mass (mean of $3474 \mathrm{~kg}$ of DM ha $\mathrm{a}^{-1}$ ) and the content of DM, neutral detergent fibre and crude protein of samples taken through grazing simulation (mean of 49.4, 76.1 and 12.9\%, respectively) were not affected by treatments. The green leaf growth rate increased $(P<0.05)$ from 12.1 to $20.5 \mathrm{~kg}$ of $D M$ $h^{-1}$ day $^{-1}$ and stocking rate decreased $(P<0.05)$ from 1178 to $609 \mathrm{~kg}$ of $B W \mathrm{ha}^{-1}$ when GLAL increased from 3.2 to $9.5 \%$ of $B W$. Animal production per area was in average negative (mean $B W$ gain of $-551 \mathrm{~g} \mathrm{ha}^{-1}$ day $^{-1}$ ), highly variable (variation coefficient of 280\%) and not affected by treatments. Individual animal performance was affected by interaction between treatments and animal breed $(P<0.05)$. Daily $B W$ gain by Lacaune-crossed wethers was positive $\left(34 g_{\text {day }}^{-1}\right)$ and higher $(P<0.05)$ for the highest allowance, null for the lowest allowance

IDepartamento de Zootecnia, Universidade Federal de Santa Maria (UFSM), 97105-900, Santa Maria, RS, Brasil. E-mail: kozloski@smail.ufsm.br. *Autor para correspondência. 
and negative $\left(-62 \mathrm{~g}\right.$ day $\left.^{-1}\right)$ for the intermediary allowance treatment. Differently, the daily $B W$ gain by Texel $\times$ Ile de France wethers was negative and similar for all treatments (mean of $-18 \mathrm{~g}$ day $^{-1}$ ). Independently of animal breeding, the daily grazing time was similar for the lowest and highest forage allowance treatments whereas it was lower for the intermediary level of forage allowance $(P<0.05)$. In conclusion, the Cynodon dactylon var. dactylon pasture showed a low productive potential, mainly when the GLAL was similar or lower than $6 \%$ of animal BW. However, results also indicate that pasture structure and animal breed interact with forage allowance to determine animal performance.

Key words: Cynodon dactylon var. dactylon, ingestive bahaviour, intake, live weight gain, tropical grass, growth rate.

\section{INTRODUÇÃO}

As pastagens constituem o principal e menos oneroso recurso forrageiro utilizado para a produção de ruminantes no Brasil, sendo que uma ampla variedade de espécies forrageiras - naturais e cultivadas - estão presentes nos sistemas de produção ou disponíveis para comercialização. O Cynodon dactylon var. dactylon é uma gramínea perene tropical usualmente classificada como invasora cosmopolita, mas apresenta algumas características positivas do ponto de vista forrageiro, incluindo grande capacidade de propagação e de adaptação a diferentes condições de solo e clima, e alta resistência ao pastejo (LIMA \& VILELA, 2005). Contudo, informações técnicocientíficas sobre o potencial nutricional do Cynodon sob pastejo são mínimas e, particularmente do Cynodon dactylon var. dactylon, são inexistentes.

$\mathrm{O}$ manejo adequado das pastagens deve contemplar o equilíbrio entre o rendimento e a qualidade da forragem produzida, simultaneamente à produção ótima por animal e por área, numa perspectiva sustentável (COLEMAN, 1992). A oferta de forragem interfere simultaneamente em todos estes aspectos e é um dos critérios recomendados para definir o manejo das pastagens. CARNEVALLI et al. (2001b) concluiram que a oferta ótima de uma pastagem de Tifton 85 (Cynodon spp.) para ovinos deveria ser em torno de 5 a $6 \mathrm{~kg}$ de matéria seca (MS) $100 \mathrm{~kg}^{-1}$ de peso corporal (PC). No entanto, uma vez que as características anatômicas e fisiológicas das diversas espécies de pastagens são diferentes, não é conclusivo se essa oferta seria adequada para outras espécies de Cynodon ou em todas as situações. Além disso, a interação planta-animal e o desempenho produtivo do sistema pastoril são afetados também pelo potencial genético dos animais, o qual interfere nas exigências nutricionais e, desse modo, pode interferir no comportamento ingestivo (CARVALHO \& MORAIS, 2005).
O presente estudo foi conduzido para avaliar o efeito do nível de oferta de forragem sobre os atributos de uma pastagem de Cynodon dactylon var. dactylon manejada sob pastejo contínuo e sobre o desempenho produtivo de ovinos de dois grupos genéticos distintos.

\section{MATERIAL E MÉTODOS}

O experimento foi conduzido de 15 de janeiro a 7 de março de 2008 na região fisiográfica denominada Depressão Central do estado do Rio Grande do Sul (altitude de 95m, latitude $29^{\circ} 43^{\prime} \mathrm{S}$ e longitude $53^{\circ} 42^{\prime} \mathrm{O}$ ). $\mathrm{O}$ solo pertence à unidade de mapeamento São Pedro, classificado como Argissolo Vermelho Distrófico Arenico (EMBRAPA, 1999), que se caracteriza por solos profundos, avermelhados, com textura superficial arenosa, friáveis e bem drenados. Foi testado o efeito de três níveis de oferta de lâminas foliares verdes (OFLV) de Cynodon dactylon var. dactylon (3, 6 e 9kg de MS $100 \mathrm{~kg}^{-1} \mathrm{PC}$ ) sobre os atributos da pastagem e sobre o desempenho produtivo de ovinos de dois grupos genéticos distintos.

A pastagem utilizada no estudo já estava previamente estabelecida e era utilizada rotineiramente sob pastejo por ovinos. Aárea experimental incluiu nove piquetes de aproximadamente 0,18 hectares (ha) cada, além de uma área adicional de 1,5ha para permanência de animais reguladores. Os tratamentos foram distribuídos nos piquetes num delineamento ao acaso. Previamente ao início do experimento foi feita uma adubação de cobertura com cerca de $280 \mathrm{~kg} \mathrm{ha}^{-1} \mathrm{de}$ NPK (05-20-20). Além disso, foram feitas duas aplicações em cobertura de $50 \mathrm{~kg} \mathrm{ha}^{-1}$ de nitrogênio na forma de uréia, uma no início de cada período experimental.

Foram utilizados 36 borregos, machos não castrados, sendo 18 animais provenientes do cruzamento alternado entre as raças Texel e Ile de France, e 18 provenientes do cruzamento entre as raças Lacaune (1/2), Texel (1/4) e Ile de France (1/4). Em cada piquete foram alocados quatro animais-teste, sendo dois de cada grupo genético. Os animais receberam uma dose de vermífugo uma semana antes do início do experimento e, ao longo do experimento, o controle de endoparasitos foi feito pelo método FAMACHA de MALAN \& VAN WYK (1992). O sistema de manejo foi de lotação contínua com carga variável, conforme metodologia proposta por MOTT \& LUCAS (1952). Os animais foram pesados no início, aos 28 dias e no final do experimento, após jejum prévio de sólidos e líquidos de aproximadamente 14 horas.

As avaliações dos atributos da pastagem foram feitas no início, aos 28 dias e no final do experimento. As massas de forragem total e de lâminas 
foliares verdes foram estimadas pelo método visual com dupla amostragem (MANNETJE, 2000). Foram feitas vinte estimativas visuais por piquete utilizando-se quadrados de $0,0625 \mathrm{~m}^{2}$, sendo que em cinco delas a biomassa de pastagem contida no interior do quadrado foi cortada rente ao solo, pesada e amostrada. Estas amostras foram então compostas por piquete, das quais foram retiradas duas sub-amostras. Uma sub-amostra foi seca em estufa com ventilação forçada a $55^{\circ} \mathrm{C}$ durante pelo menos três dias para estimativa da massa total da pastagem. A outra foi utilizada para separação manual dos componentes: lâmina de folha, colmo + bainha e material senescente, os quais também foram secos em estufa com ventilação forçada a $55^{\circ} \mathrm{C}$.

Com a utilização de duas gaiolas de exclusão ao pastejo em cada piquete (GARDNER, 1986) foi determinada a taxa de acúmulo diária das lâminas foliares verdes (TAFV). Para tal, a biomassa de forragem contida no interior da gaiola e em igual área do lado de fora da gaiola foi cortada e processada como descrito anteriormente. A TAFV foi obtida pela diferença entre a massa de folhas presente dentro da gaiola no final do período menos a massa de folhas existente fora da gaiola no início do período, dividida pelo número de dias do período. A carga animal (CA) em cada tratamento foi ajustada noinício e aos 28 dias do experimento, sendo calculada como: CA $\left(\mathrm{kg}\right.$ de $\left.\mathrm{PC} \mathrm{ha}^{-1}\right)=\left[\left(\left(\mathrm{TAFV} \times \mathrm{n}^{\circ}\right.\right.\right.$ dias período $)+\mathrm{MS}$ de folhas verdes $) /\left(\mathrm{n}^{\circ}\right.$ dias período $\left.\left.\times 100\right)\right]$ /oferta pretendida. Para ajuste da CAnoinício do experimento a TAFV foi estimada em 16kg de MS ha- ${ }^{-1} \mathrm{dia}^{-1}$.

Amostras de simulação de pastejo de cada animal-teste foram coletadas de cada piquete aos 28 dias do período experimental. Um indivíduo em cada piquete observou o ato de apreensão da pastagem de cada animal teste durante trinta minutos, no período da manhã, coletando manualmente em cinco amostras representativas do material apreendido pelos animais. Estas amostras foram secas em estufa com ventilação forçada a $55^{\circ} \mathrm{C}$, moídas, compostas por piquete, e armazenadas para posterior análise. Para estimar a excreção fecal, cada animal-teste recebeu via oral diariamente, a partir do $16^{\circ}$ dia do primeiro período experimental e durante 12 dias, uma cápsula gel contendo $1 \mathrm{~g}$ de óxido de cromo. Nos últimos quatro dias do fornecimento do indicador, amostras de fezes foram coletadas diretamente do reto a cada 12 horas, avançando 3 horas a cada dia, de modo a ter amostras a intervalos de 3 horas num período de 24 horas. Essas amostras foram compostas por animal, secas em estufa com ventilação forçada a $55^{\circ} \mathrm{C}$, moídas, e armazenadas para posterior análise. $\mathrm{O}$ teor de MS foi determinado por secagem em estufa a $105^{\circ} \mathrm{C}$ durante $8 \mathrm{~h}$, e o conteúdo de cinzas foi determinado por combustão a $600^{\circ} \mathrm{C}$ durante $3 \mathrm{~h}$. O nitrogênio total (N) foi determinado por um método Kjeldahl (Método 984.13, AOAC, 1995). O teor de proteína bruta $(\mathrm{PB})$ foi calculado como o teor de $\mathrm{N} \times 6,25$. O teor de fibra em detergente neutro (FDN) e de fibra em detergente ácido (FDA) foi analisado utilizando autoclave conforme descrito por SENGER et al. (2008). A digestibilidade in vitro da matéria seca (DIVMS) foi determinada através de método adaptado de GOERING \& VAN SOEST (1970), sendo as amostras pesadas e incubadas em saquinhos de nylon (porosidade de $50 \mu$ ) durante 48 horas e o resíduo tratado com solução detergente neutro em autoclave (SENGER et al., 2008). O teor de cromo foi determinado por espectrofotometria de absorção atômica após queima em mufla e digestão ácida (KOZLOSKI et al., 2006). A excreção fecal de MS ( $\mathrm{g} \mathrm{dia}^{-1}$ ) foi estimada como: [684/concentração fecal de cromo ( $\mathrm{mg} \mathrm{g}^{-1}$ de MS)]. O consumo de pasto foi calculado utilizando uma equação que relaciona a excreção fecal de N e FDA com o consumo de MS, desenvolvida em um ensaio de digestibilidade com ovinos mantidos em gaiolas de metabolismo e recebendo Cynodon dactylon var. dactylon como único alimento (OLIVEIRA, 2009): ConsumodeMS $\left(\mathrm{g} \mathrm{dia}^{-1}\right)=178+\left(1,73 \times\right.$ FDA fecal $\left.\left(\mathrm{g} \mathrm{dia}^{-1}\right)\right)+$ $\left(64,5 \times \mathrm{N}\right.$ fecal $\left.\left(\mathrm{g} \mathrm{dia}^{-1}\right)\right)$.

$\mathrm{O}$ comportamento ingestivo dos animaisteste foi avaliado no $15^{\circ}$ dia do período experimental, das 06h50min às 20h30min. Foram feitas anotações a cada dez minutos, por meio de observação visual (JAMIESON \& HODGSON, 1979), quando foram registradas as atividades de pastejo, ruminação e ócio.

A análise da variância dos dados foi feita utilizando-se o procedimento MIXED do programa estatístico SAS (2004), que incluiu no modelo os efeitos de oferta de forragem, grupo genético e da interação oferta de forragem $\times$ grupo genético, além do erro experimental. As médias foram comparadas pelo teste $t$ de Student.

\section{RESULTADOS E DISCUSSÃO}

Os resultados das variáveis quantitativas e qualitativas da pastagem são apresentados na tabela 1 . A OFLV real foi próximo à pretendida em cada tratamento (i.e. 3,2, 6,7 e 9,5\% do PV). A massa de forragem (média de $3474 \mathrm{~kg}$ de MS ha-1), assim como os teores de MS, FDN e PB das amostras coletadas por simulação (médias de 49,4, 76,1 e 12,9, respectivamente) não foram afetados pelos tratamentos. A DIVMS destas amostras tendeu $(\mathrm{P}=0,09)$ a ser mais alta no tratamento de maior oferta. A proporção de folhas na massa total de forragem foi mais baixa no tratamento de menor oferta comparado com o tratamento de oferta mais alta 
Tabela 1 - Variáveis estruturais da pastagem e produção animal em uma pastagem de Cynodon dactylon var. dactylon submetida a pastejo por ovinos em três níveis de oferta de forragem.

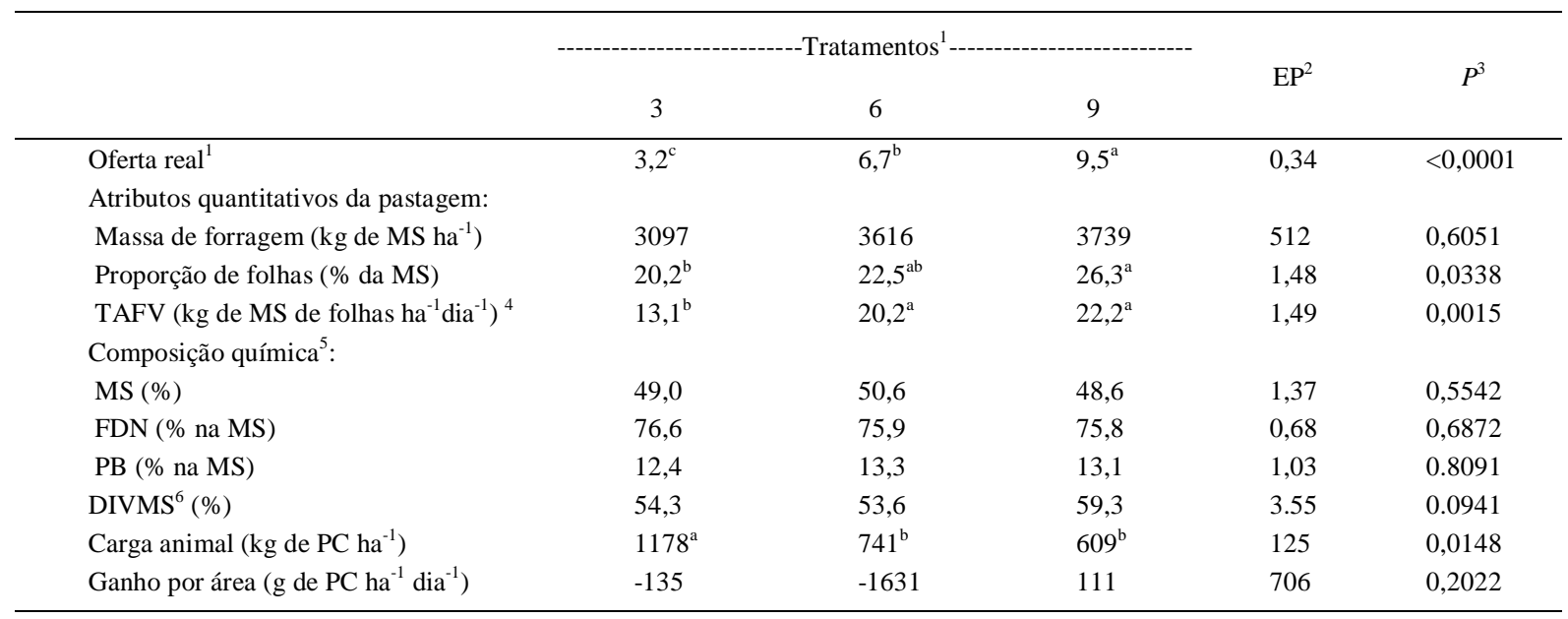

a,b: médias são diferentes pelo teste t de Student $(\mathrm{P}<0,05)$

${ }^{1} \mathrm{~kg}$ de matéria seca (MS) de lâminas foliares verdes $100 \mathrm{~kg}^{-1}$ de peso corporal ${ }^{-1}(\mathrm{PC})$.

${ }^{2}$ Erro padrão das médias, onde $\mathrm{n}=6$ por tratamento.

${ }^{3}$ Probabilidade do efeito de tratamento pelo teste F.

${ }^{4}$ Taxa de acúmulo de folhas verdes.

5 Amostras coletadas por simulação de pastejo.

${ }^{6}$ Digestibilidade in vitro da MS das amostras coletadas por simulação de pastejo.

$(\mathrm{P}<0,05)$. A proporção de folhas na forragem no tratamento com oferta intermediária não diferiu das demais. ATAFV foi mais baixa na menor oferta $(\mathrm{P}<0,05)$ e mais alta nas ofertas de 6 e 9\%, que não diferiram entre si. Usualmente o aumento da intensidade de pastejo reduz a disponibilidade de folhas verdes, a taxa fotossintética e o crescimento das pastagens (PARSONS et al., 1999). Contudo, em trabalhos similares com ovinos mantidos em pastagem de Coastcross (CARNEVALLI et al., 2001a) ou Tifton 85 (CARNEVALLI et al., 2001b) foi observado que o aumento da altura da pastagem de 50 para $200 \mathrm{~mm}$, e consequentemente o aumento da oferta de forragem total de em torno de 3 para $7 \%$ do PC, reduziu a proporção de folhas e aumentou a proporção de material morto no pasto. Adicionalmente, nos estudos de CARNEVALLI et al. (2001a e 2001b) a proporção de folhas foi menor (médias em torno de $18 \%$ vs. $23 \%$ ) enquanto a TAFV das pastagens de Coastcross ou Tifton 85 foi em torno de três a cinco vezes mais alta que a observada na pastagem de Cynodon no presente estudo. Os valores relativamente baixos de TAFV do Cynodon dactylon, assim como os altos teores de MS das folhas estão associados, pelo menos em parte, à deficiência hídrica que predominou durante a maior parte do experimento (dados não apresentados).
Como esperado, a carga animal reduziu significativamente $(\mathrm{P}<0,05)$ à medida que aumentou a oferta de forragem. A produtividade animal por área de pastagem foi em média negativa (ganho de peso vivo por área de $-551 \mathrm{~g} \mathrm{ha}^{-1} \mathrm{dia}^{-1}$ ), altamente variável (coeficiente de variação de $280 \%$ ) e, por isso, não foi afetada significativamente pelos tratamentos. Isto foi conseqüência do baixo e variável desempenho individual dos ovinos mantidos em pastejo (Tabela 2).

$\mathrm{O}$ aumento da carga animal normalmente diminui a oportunidade de escolha do pasto a ser ingerido e reduz o ganho individual dos animais em pastejo (HODGSON, 1990; COLEMAN, 1992). No estudo de CARNEVALLI et al. (2001b), por exemplo, o ganho diário de PC dos ovinos aumentou linearmente de -21g para $35 \mathrm{~g}$ quando a oferta de MS de forragem total aumentou de 2,4 para 6,6\% do PC, mesmo que a proporção de folhas tenha diminuído. No entanto, no presente estudo houve interação entre grupo genético versus oferta $(\mathrm{P}<0,05)$ sobre o consumo de MS e sobre o desempenho animal. O consumo de MS pelos ovinos cruzados com Lacaune foi similar em todos os tratamentos, mas o ganho de peso desses animais foi positivo e mais alto na maior oferta, nulo na menor oferta e negativo na oferta intermediária de pasto $(\mathrm{P}<0,05)$. Diferentemente, o consumo de MS pelos 
Tabela 2 - Desempenho de cordeiros de dois grupos genéticos em pastagem de Cynodon dactylon var. dactylon submetidos a três níveis de oferta de lâminas foliares verdes.

\begin{tabular}{|c|c|c|c|c|c|}
\hline & 3 & 6 & 9 & $\mathrm{EP}^{1}$ & $P^{2}$ \\
\hline Peso inicial (kg) & 24,4 & 26,2 & 24,0 & 1,11 & 0,3700 \\
\hline Peso final (kg) & 25,3 & 22,8 & 25,8 & 1,01 & 0,1230 \\
\hline Ganho médio diário (g) & $0^{\mathrm{b}}$ & $-62^{c}$ & $34^{\mathrm{a}}$ & 12,5 & 0,0003 \\
\hline Consumo de MS (g dia $\left.{ }^{-1}\right)$ & 911 & 910 & 869 & 42,3 & 0,7323 \\
\hline Peso inicial (kg) & 21,1 & 23,1 & 22,4 & 1,20 & 0,5054 \\
\hline Peso final (kg) & 19,6 & 22,1 & 21,0 & 1,36 & 0,4208 \\
\hline Ganho médio diário (g) & -29 & -17 & -8 & 15,1 & 0,6450 \\
\hline Consumo de MS $\left(\mathrm{g} \mathrm{dia}^{-1}\right)$ & $731^{\mathrm{b}}$ & $830^{\mathrm{ab}}$ & $897^{\mathrm{a}}$ & 46,9 & 0,0712 \\
\hline
\end{tabular}

a,b,c: médias são diferentes pelo teste t de Student $(\mathrm{P}<0,05)$

${ }^{1} \mathrm{~kg}$ de matéria seca (MS) de lâminas foliares verdes $100 \mathrm{~kg}^{-1}$ de peso corporal.

${ }^{2}$ Erro padrão das médias, onde $\mathrm{n}=6$ por tratamento.

${ }^{3}$ Probabilidade do efeito de tratamento pelo teste F.

animais Texel $\times$ Ile de France foi diretamente afetado pela oferta de forragem $(\mathrm{P}<0,05)$ enquanto o ganho de peso foi negativo e similar em todos os tratamentos (média de - $18 \mathrm{~g}$ dia $^{-1}$ ).

Os resultados de desempenho dos ovinos cruzados com Lacaune foram mais coerentes com as observações de comportamento diário dos animais (Tabela 3) do que os Texel $\times$ Ile de France. Em ambas espécies animais, o tempo de pastejo foi menor no tratamento de oferta intermediária de forragem $(\mathrm{P}<0,05)$. As demais variáveis comportamentais não foram afetadas pelos tratamentos. Em média, os ovinos utilizaram $47 \%$ do tempo pastejando, $33 \%$ ruminando e $20 \%$ em outras atividades. Em média, os animais apresentaram uma taxa de 26 bocados por minuto.
Como previamente evidenciado em outros estudos (CARVALHO et al., 2001; RUTTER, 2006; BOVAL et al., 2007) os resultados do presente estudo indicam que não somente a oferta, mas também a distribuição das folhas na estrutura do dossel e a capacidade individual de cada animal em acessar e ingerir forragem de qualidade interfere no consumo e desempenho de ovinos a pasto.

\section{CONCLUSÃO}

Em uma condição de deficiência hídrica, a pastagem Cynodon dactylon var.dactylon apresentou um baixo potencial produtivo, principalmente quando a oferta de matéria seca de lâminas foliares verdes foi

Tabela 3 - Tempo diário (minutos) de pastejo, ócio e ruminação por ovinos mantidos em uma pastagem de Cynodon dactylon var. dactylon a três níveis de oferta de forragem.

\begin{tabular}{lccccc}
\hline & & & & \\
& 3 & 6 & & $E^{2}$ & $P^{3}$ \\
\hline Tempo de pastejo & $267^{\mathrm{a}}$ & $217^{\mathrm{b}}$ & $280^{\mathrm{a}}$ & 11,7 & 0,0014 \\
Tempo de ócio & 120 & 124 & 85 & 17,5 & 0,2370 \\
Tempo de ruminação & 180 & 191 & 170 & 11,5 & 0,4483 \\
\hline
\end{tabular}

a,b: médias são diferentes pelo teste $t$ de Student $(\mathrm{P}<0,05)$

${ }^{1} \mathrm{~kg}$ de matéria seca (MS) de lâminas foliares verdes $100 \mathrm{~kg}^{-1}$ de peso corporal.

${ }^{2}$ Erro padrão das médias, onde $\mathrm{n}=12$ por tratamento.

${ }^{3}$ Probabilidade do efeito de tratamento pelo teste $\mathrm{F}$. 
igual ou inferior a $6 \%$ do peso corporal dos animais. Contudo, os resultados indicam também que a estrutura da pastagem e o potencial genético interagem com a oferta de forragem para determinar o desempenho de animais a pasto.

\section{REFERÊNCIAS}

AOAC (ASSOCIATION OF OFFICIAL ANALYTICAL CHEMISTS). Official methods of analysis. 16.ed. Gaithersburg, 1997. 1298p.

BOVAL, M. et al. Effect of structure of a tropical pasture on ingestive behaviour, digestibility of diet and daily intake by grazing cattle. Grass and Forage Science, v.62, p.44-54, 2007. Disponível em: <http://onlinelibrary.wiley.com/doi/ 10.1111/j.1365-2494.2007.00560.x/abstract>. Acesso em: 22 ago. 2012. doi: 10.1111/j.1365-2494.2007.00560.x

CARNEVALLI, R.A. et al. Desempenho de ovinos e respostas de pastagens de Coastcross submetidas a regimes de desfolha sob lotação contínua. Pesquisa Agropecuária Brasileira, v.36, p.919-927, 2001a. Disponível em: <http://www.scielo.br/ scielo.php ? script $=\mathrm{sci}$ art text \& pid=S $0100-$ 204X2001000600010>. Acesso em: 22 ago. 2012. doi: $10.1590 / \mathrm{S} 0100-204 \mathrm{X} 2001000600010$

CARNEVALLI, R.A. et al. Desempenho de ovinos e respostas de pastagens de tifton 85 (Cynodon spp.) sob lotação contínua. Scientia Agricola, v.58, p.7-15, 2001b. Disponível em: <http:/ $/$ www.scielo.br/scielo.php?script=sci_arttext\&pid=S010390162001000100002>. Acesso em: 22 ago. 2012. doi: 10.1590/ S0103-90162001000100002.

CARVALHO, P.C.F.; MORAES, A. Comportamento ingestivo de ruminantes: bases para o manejo sustentável do pasto. In: MANEJO SUSTENTÁVEL EM PASTAGEM, 2005, Maringá, PR. Anais... Maringá: UFPR, 2005. p.1-20.

CARVALHO, P.C.F. et al. Importância da estrutura da pastagem na ingestão e seleção de dietas pelo animal em pastejo. In: REUNIÃO ANUAL DA SOCIEDADE BRASILEIRA DE ZOOTECNIA: A Produção animal na visão dos brasileiros, Piracicaba, 2001. Anais... Piracicaba: SBZ, 2001. p.853-871.

COLEMAN, S.W. Plant-animal interface. Journal of Production Agriculture, v.5, p.7-13, 1992.

EMBRAPA (EMPRESA BRASILEIRA DE PESQUISA AGROPECUÁRIA). Centro Nacional de Pesquisa de Solos. Sistema Brasileiro de classificação de solos. Brasília: EMBRAPA, 1999. 412p.

GARDNER, A.L. Técnicas de pesquisa em pastagens e aplicabilidade de resultados em sistemas de produção. Brasil: IICA, 1986. p.197.

GOERING, H.K.; VAN SOEST, P.J. Forage fiber analysis: apparatus reagents, procedures and some applications. Washington, D.C.: USDA, 1970. 20p. (Agricultural Handbook, n.379).

HODGSON, J. Grazing management: science into practice. New York: John Wiley \& Sons, 1990. 203p.

JAMIESON, W.S.; HODGSON, J. The effect of daily herbage allowance and sward characteristics upon the ingestive behavior of calves under strip-grazing management. Grass and Forage
Science, v.34, p.261-271, 1979. Disponível em: <http:// onlinelibrary.wiley.com/doi/10.1111/j.1365-2494.1979.tb01478.x/ abstract>. Acesso em: 22 ago. 2012. doi: 10.1111/j.13652494.1979.tb01478.x.

KOZLOSKI, G.V. et al. Uso do óxido de cromo como indicador da excreção fecal de bovinos em pastejo: variação das estimativas em função do horário de amostragem. Ciência Rural, v.36, p.599-603, 2006. Disponível em: <http://www.scielo.br/ scielo.php? script =sci_art text \& pid=S 0103 84782006000200037>. Acesso em: 22 ago. 2012. doi: 10.1590/ S0103-84782006000200037.

LIMA, J.A.; VILELA, D. Formação e manejo de pastagens de Cynodon. In: VILELA, D. et al. Cynodon: forrageiras que estão revolucionando a pecuária brasileira. Juiz de Fora: EMBRAPA, 2005. p.11-32.

MALAN, F.S.; VAN WYK, J.A. The packed cell volum and color of the conjunctivae as aids for monitorin Haemonchus contortus infestations in sheep. In: BIENNIAL NATIONAL VETERINARY CONGRESS, 1992, Grahamstown, África do Sul. Anais... Grahamstown: South African Veterinary Association, 1992. V.1, p.139.

MANNETJE, L.t'. Measuring biomass of grassland vegetation. In: MANNETJE, L.t'; JONES, R.M. Field and laboratory methods for grassland and animal production research. Cambridge: CABI, 2000. p.151-178.

MOTT, G.O.; LUCAS, H.L. The design, conduct and interpretation of grazing trials in cultivated and improved pastures. In: INTERNATIONAL GRASSLANDS CONGRESS, 6., 1952, State College. Proceedings... State College: Pennsylvania, State College, 1952. p.1380-1385.

NEVES, F.P. et al. Estratégias de manejo da oferta de forragem para recria de novilhas em pastagem natural. Revista Brasileira de Zootecnia, v.38, p. 1532-1542, 2009. Disponível em: <http:/ /www.scielo.br/scielo.php?script=sci_arttext \&pid=S151635982009000800018>. Acesso em: 22 ago. 2012. doi: 10.1590/ S1516-35982009000800018.

OLIVEIRA, L. Métodos em nutrição de ruminantes: estimativa do consumo através de índices fecais e estimativa de síntese microbiana ruminal. 2009. 78f. Dissertação (Mestrado em Zootecnia) - Programa de Pós-graduação em Zootecnia, Universidade Federal de Santa Maria, RS. Disponível em: <http:/ /w3.ufsm.br/ppgz/dissertacoes3.html>. Acesso em: 22 ago. 2012.

PARSONS, A.J. et al. Dynamics of heterogeneity in a grazed sward. In: GRASSLAND ECOPHYSIOLOGY AND GRAZING ECOLOGY, 1999, Curitiba, PR. Anais... Curitiba: Universidade Federal do Paraná, 1999. p.187-214.

RUTTER, S.M. Diet preference for grass and legumes in free-ranging domestic sheep and cattle: current theory and future application. Applied Animal Behaviour Science, v.97 p.17-35, 2006. Disponível em: <http://www.journals.elsevierhealth.com/periodicals/ applan/article/S0168-1591\%2805\%2900397-7/abstract>. Acesso em: 22 ago. 2012. doi: 10.1016.applanim.2005.11.016.

SENGER, C.C.D. et al. Evaluation of autoclave procedures for fibre analysis in forage and concentrate feedstuffs. Animal Feed Science and Technology, v.146, p.169-174, 2008. Disponível em: <http://dx.doi.org/10.1016/j.anifeedsci.2007.12.008>. Acesso em: 22 ago. 2012. doi: 10.1016/j.anifeedsci.2007.12.008.

SAS (STATISCAL ANALYSIS SYSTEM). SAS/STAT ${ }^{\circledR}$ User's $^{\text {'s }}$ guide, Version 9.1. Cary, NC, 2004. 5136p. 